\title{
A comparative study of electrochemical, spectroscopic and structural properties of phenyl, thienyl and furyl substituted ethylenes
}

\author{
Lucia Viglianti, ${ }^{[b] \dagger}$ Francesca Villafiorita-Monteleone,${ }^{[\mathrm{c}]}$ Chiara Botta, ${ }^{[\mathrm{c}]}$ Patrizia R. Mussini, ${ }^{[\mathrm{b}]}$ Emanuele \\ Ortoleva, ${ }^{[b]}$ Silvia Cauteruccio, ${ }^{[b]}$ Emanuela Licandro, ${ }^{[b]}$ and Clara Baldoli ${ }^{[a]}$
}

\begin{abstract}
: a detailed electrochemical and photophysical comparative study of three parallel series of phenyl, thienyl and furyl substituted ethylenes has been carried out, implemented by the computational calculation of selected terms. Relationships have been highlighted between molecular structure (number and type of aromatic rings) and important functional properties (in particular, electronic features and oligomerization ability). Interestingly, some of the studied heteroaryl-ethylenes show emission in the solid state displaying an aggregation-induced emission behavior.
\end{abstract}

\section{Introduction}

Luminogenic molecules with aggregation-induced emission (AIE) properties have drawn increasing research interest as components for the development of functional materials ${ }^{1}$ in a broad range of fields including optics, electronics, energy and bioscience. The AIE effect was discovered by B. Z. Tang ${ }^{2}$ and refers to a photophysical phenomenon shown by a group of luminogenic molecules that are not emissive in solution because of rotational and vibrational deactivation pathways, but become highly luminescent when clustered as aggregates in poor solvents or solid states, where radiative relaxation is allowed. ${ }^{3}$ A simple molecule such as tetraphenylethylene (TPE, Figure 1) shows an intense AIE effect, and a large number of TPE derivatives have been used for the construction of different luminescent materials. ${ }^{4}$ On the contrary, heteroaromatic TPE analogs containing electron-rich five member rings such as thiophene or furan have been scarcely considered so far, ${ }^{5}$ and a detailed study of their electro-optical properties was overdue. In a previous paper we reported the synthesis, photophysics and electrochemistry of di- and tetra-(2-thienyl)ethylene (DTE and TTE, figure 1) compared to their phenyl analogs (DPE and TPE). ${ }^{6}$ From this preliminary study we found that TTE was a

\footnotetext{
[a] Dr. C. Baldoli

CNR - Istituto di Scienze e Tecnologie Molecolari

Via C. Golgi 19. 20133 Milano, Italy.

E-mail: clara.baldoli@istm.cnr.it

[b] L.Viglianti, P. R. Mussini, E. Ortoleva, S. Cauteruccio, E. Licandro. Dipartimento di Chimica, Università degli Studi di Milano

Via C. Golgi 19. 20133 Milano, Italy.

[c] F. Villafiorita-Monteleone, C. Botta

CNR - Istituto per lo Studio delle Macromolecole.Via A. Corti 12. 20133 Milano, Italy.

$\dagger \quad$ Present address: The Hong Kong University of Science and Technology, Departement of Chemistry Clear Water Bay, Kowloon, Hong Kong. China
}

promising AIE active molecule, which is nearly non-luminescent in concentrated or even saturated dichloromethane solution, but emissive in rigid matrixes or in the solid state. This attractive finding prompted us to perform a systematic investigation on the relationships between molecular structure and electrochemical and optoelectronic properties in the three families of substituted ethylenes: namely thienyl- and furyl derivatives, plus phenyl ones in the same conditions for sake of comparison (Figure 1). In this paper we report the results of a combined electrochemical, spectroscopic and computational study, highlighting the effect of the aromatic substituent type and number on the overall electronic properties and oligomerization ability of heteroaryl substituted ethylene series.

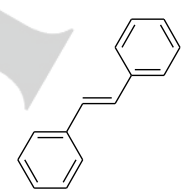<smiles></smiles>

DTE

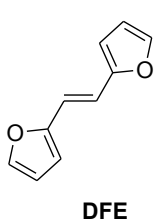

DFE

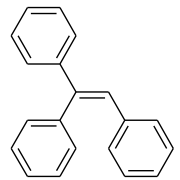

TrPE

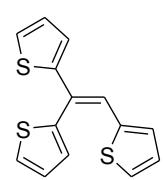

TrTE

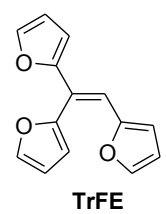

TrFE

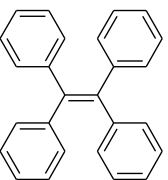

TPE

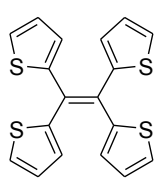

TTE

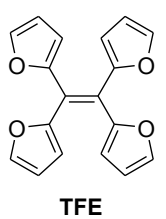

Figure 1. (Hetero)arylethylene series

\section{Results and Discussion}

\section{Synthesis}

Of the nine molecules object of the present paper (Figure 1), phenyl derivatives, DTE and DFE are commercially available. TrTE is a new molecule while TrFE has been mentioned in the literature ${ }^{7}$ but not fully characterized. TTE is a known molecule ${ }^{6,8}$ while TFE, which was under investigation in our group, ${ }^{9}$ has been recently reported in the in literature. ${ }^{10}$ For the purposes of this work, we synthesized DFE, TFE, and TTE from the corresponding carbonyl compounds $1-3,{ }^{11}$ (Scheme 1 ) using a 
slight modification of the reported McMurry conditions: pyridine was not used as additive in the reaction, and the title compounds were obtained in moderate yields, after chromatographic purification.<smiles>[R]C(=O)c1[X]ccc1</smiles>

1: $X=O ; R=H$

3: $X=S ; R=2$-thienyl
2: $X=O ; R=2$-furyl

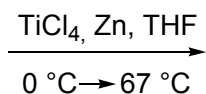

DFE: $X=O ; R=H, 66 \%$
TFE: $X=O ; R=2$-furyl, $49 \%$ TTE: $X=S ; R=2$-thienyl, $55 \%$
Scheme 1. Synthesis of DFE, TTE and TrFE

The trisubstituted derivatives TrTE and TrFE were prepared through a Pd-catalyzed cross-coupling reaction between gemdibromo derivatives ${ }^{12} \mathbf{4}$ or $\mathbf{5}$ and 2-thienyl or 2-furyl dioxaborolanes 6 or 7 . (Scheme 2) The reaction was carried out in refluxing $\mathrm{THF} / \mathrm{H}_{2} \mathrm{O}$ solution, in the presence of $\mathrm{K}_{2} \mathrm{CO}_{3}$; the target compounds were obtained, after chromatographic purification, in $70 \%$ and $30 \%$ yield respectively and were completely characterized.

\section{Electrochemical studies}

The redox properties and electropolymerization ability of all (hetero)arylethylenes, shown in Figure 1, have been studied by cyclic voltammetry (CV) both in acetonitrile (ACN) and in dichloromethane (DCM) as solvents. A synopsis of normalized $\mathrm{CV}$ features for the whole series, in $\mathrm{ACN}$ as solvent, sorted by number of heterocyclic rings at the double bond, is shown in Figure 2. Synopses sorted by type of heterocycle are available in the Supporting Information for both the ACN and the DCM case (Figures EC1 and EC2, respectively). The two chosen solvents not only differ in polarity (ACN more polar than DCM) but also in the available potential window, as DCM undergoes electroreduction at a significantly less negative potential than ACN (i.e. -2.2 vs SCE), thus preventing the observation of the first reduction peaks of the less conjugated molecules (e.g. diand tri- substituted heteroarylethenes).

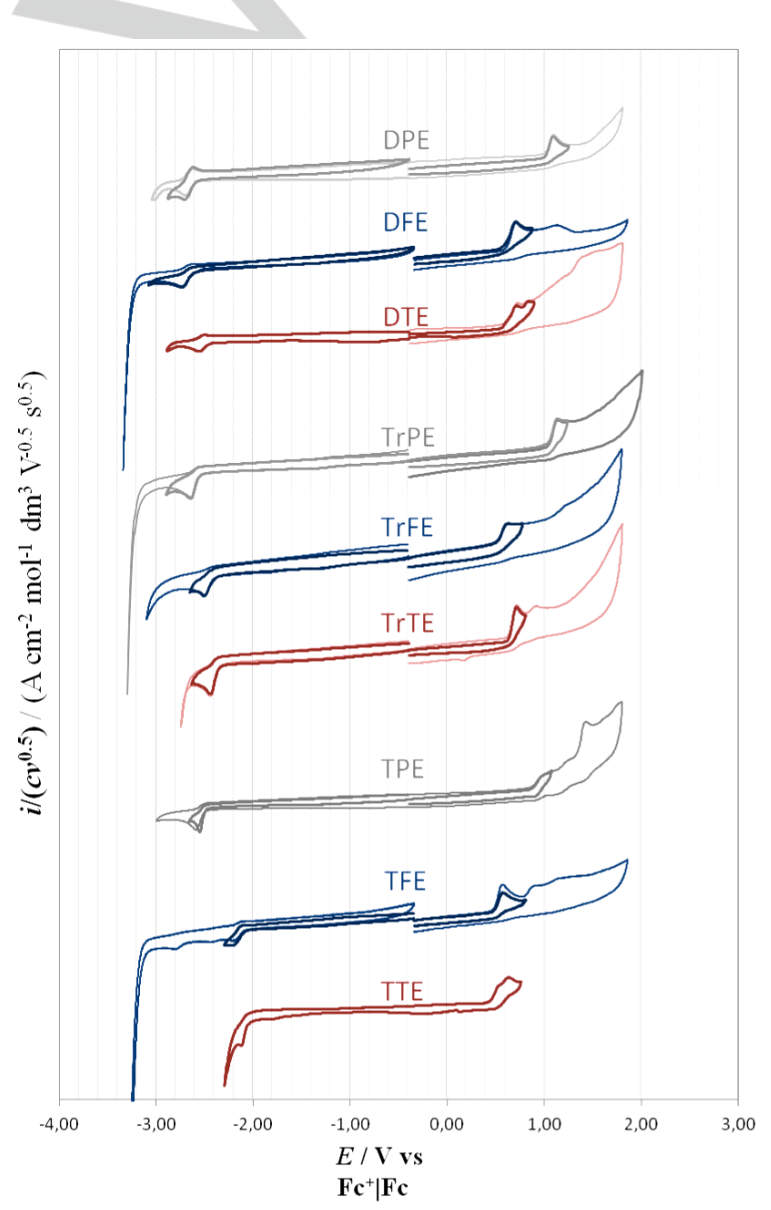

Figure 2. Normalized $\mathrm{CV}$ patterns for the investigated (hetero)arylethylenes (sorted by number of rings), in $\mathrm{ACN}+0.1 \mathrm{M} \mathrm{TBAPF}_{6}$, at $0.2 \mathrm{~V} / \mathrm{s}$ scan rate.

Normalized CV patterns at increasing scan rates are also reported in the SI (Figures EC3, EC4, EC5), since they provide information about two important features in the perspective of device applications, i.e.: a) facility of the electron transfer (ET): the larger the peak potential shift with increasing scan rate, the 
higher the ET activation barrier, while constant peak potential with scan rate points to an extremely facile ET ("electrochemically reversible" process) b) chemical reversibility of the ET process: i.e. chemical stability of the ET product, so that it can be reconverted into the reacting molecule by inverting the potential scan: this is accounted for by the presence of a symmetrical return peak. The presence of a competitive chemical follow-up process is evidenced by this return peak gradually disappearing upon reducing the potential scan rate.

As it can be seen in Figure 2, most first oxidation and reduction processes appear chemically irreversible at $0.2 \mathrm{~V} / \mathrm{s}$, consistently with the involvement of the radical-ion ET product in a subsequent chemical process. However, in several cases reversibility improves with increasing scan rate (SI, Figures EC3EC5), particularly in the phenyl cases, showing a rather slow chemical process following the electron transfer. Furthermore, in most cases (either chemically reversible or irreversible) the peak potential is nearly constant with scan rate, pointing to a rather facile electron transfer.

Values of onset and peak potentials for first oxidations and reductions in ACN are collected in Table 1 (data in DCM are also available in the $\mathrm{SI}$ ) together with the corresponding HOMO and LUMO energies and energy gaps $\left(E_{\mathrm{g}}\right)$, calculated from the equations reported in the $\mathrm{SI},{ }^{13}$ ultimately based on the estimated absolute value for the normal hydrogen electrode (NHE). ${ }^{14}$

\begin{tabular}{ccccccc}
\multicolumn{5}{c}{ Table 1. Key CV data for the (hetero)arylethylene series in ACN. ${ }^{\text {[a] }}$} \\
\hline Compd. & $\begin{array}{c}E_{\text {ox }}[\mathrm{V}]^{[\mathrm{b}]} \\
\text { onset/max }\end{array}$ & $\begin{array}{c}E_{\text {red }}[\mathrm{V}]^{[\mathrm{b}]} \\
\text { onset/max }\end{array}$ & $\begin{array}{c}E_{\text {Homo }}[\mathrm{eV}] \\
\text { onset/max }\end{array}$ & $\begin{array}{c}E_{\mathrm{LUMo}}[\mathrm{eV}] \\
\text { onset/max }\end{array}$ & $\begin{array}{c}E_{\mathrm{g}}[\mathrm{eV}] \\
\text { onset/max }\end{array}$ \\
\hline DPE & $1.01 / 1.10$ & $-2.57 /-2.70$ & $-5.81 /-5.90$ & $-2.23 /-2.10$ & $3.58 / 3.80$ \\
DFE & $0.57 / 0.71$ & $-2.61 /-2.72$ & $-5.37 /-5.51$ & $-2.19 /-2.08$ & $3.18 / 3.43$ \\
DTE & $0.61 / 0.72$ & $-2.44 /-2.54$ & $-5.41 /-5.52$ & $-2.36 /-2.26$ & $3.05 / 3.26$ \\
TrPE & $1.03 / 1.14$ & $-2.64 /-2.54$ & $-5.83 /-5.94$ & $-2.16 /-2.26$ & $3.67 / 3.68$ \\
TrFE & $0.52 / 0.61$ & $-2.40 /-2.49$ & $-5.32 /-5.41$ & $-2.40 /-2.31$ & $2.92 / 3.10$ \\
TrTE & $0.63 / 0.72$ & $-2.34 /-2.44$ & $-5.43 /-5.52$ & $-2.46 /-2.36$ & $2.97 / 3.16$ \\
TPE & $0.88 / 0.97$ & $-2.49 /-2.55$ & $-5.68 /-5.77$ & $-2.31 /-2.25$ & $3.37 / 3.52$ \\
TFE & $0.47 / 0.57$ & $-2.08 /-2.19$ & $-5.27 /-5.37$ & $-2.72 /-2.61$ & $2.55 / 2.76$ \\
TTE & $0.44 / 0.54,0.64$ & $-2.05 /-2.12$ & $-5.24 /-5.34$ & $-2.75 /-2.68$ & $2.49 / 2.66$
\end{tabular}

[a] A more detailed table of CV data, also in DCM as solvent, are reported in SI. [b] vs $\left(\mathrm{Fc}^{+} / \mathrm{Fc}\right)$.

It is interesting to compare such results with literature data (collected in Table 2) for linear oligo-phenyls, -thiophenes and furans, considered as benchmark compounds ${ }^{15}$. A visual representation of HOMO and LUMO energy levels is provided by figure 3 .

Within each series (phenyl-, thienyl- and furylethylenes), both oxidation and reduction processes become easier and take place at less extreme potentials with increasing ring number, as expected. This is of course consistent with the increase in effective conjugation. Comparing the three series at constant number of aromatic rings, both thiophene- and furan-based systems have smaller gaps with respect to the corresponding phenyl-based ones, pointing to higher effective conjugation for the heteroaryl-based systems. In furyl ethylenes a more remarkable LUMO decrease with increasing ring number is observed.

Table 2 Electrochemical literature data ${ }^{15}$ for linear oligo(hetero)aryls considered as benchmarks. ${ }^{\text {[a] }}$

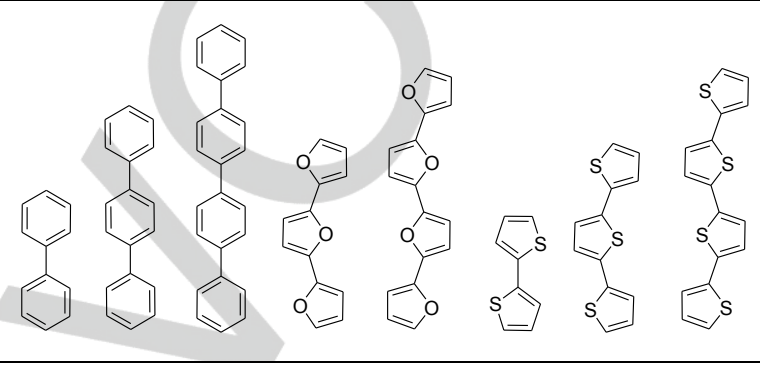

\begin{tabular}{l|cccccccc}
\hline \multicolumn{2}{c}{ P2 } & P3 & P4 & F3 & F4 & T2 & T3 & T4 \\
\hline $\begin{array}{l}E_{\text {HOMO }} \\
\max [\mathrm{eV}]\end{array}$ & -6.29 & -6.03 & -5.90 & -5.37 & -5.24 & -5.68 & -5.34 & -5.23 \\
$\begin{array}{l}E_{\text {LUMO }} \\
\max [\mathrm{eV}]\end{array}$ & -1.71 & -1.96 & -2.09 & - & - & -1.96 & -2.30 & -2.46 \\
$E_{\mathrm{g}}$ & & & & & & & & \\
$\max [\mathrm{eV}]$ & 4.58 & 4.07 & 3.81 & - & - & 3.72 & 3.04 & 2.77
\end{tabular}

[a] reported to $\mathrm{Fc}+/ \mathrm{Fc}$ reference redox couple

As expected, heterocyclic derivatives also appear much electron richer with respect to phenyl systems; in fact their energy gaps $\left(E_{g}\right)$ are not only smaller but also displaced towards higher energies (favoured oxidation, unfavoured reduction, with a less positive oxidation peak and a more negative reduction one).

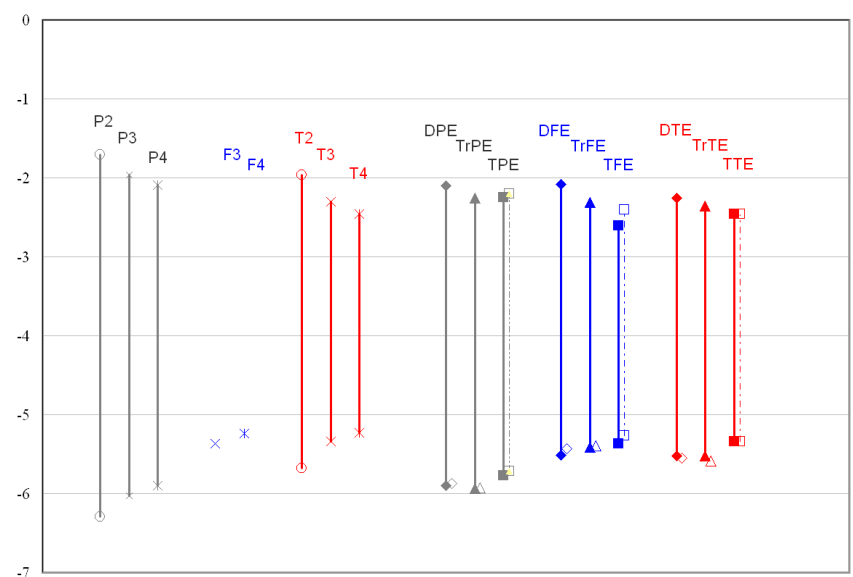

Figure 3. Electrochemical HOMO and LUMO energy levels and gaps for the whole ethylene series, obtained in ACN (full symbols, solid lines) or DCM (empty symbols, dotted lines) plus literature values for the benchmark compounds. 
It is also useful to compare ethylene derivatives with linear oligoheteroaryls as benchmarks (Table 2 and figure 3 ).

DPE has a quite smaller gap respect to $P 2$ (i.e. much higher effective conjugation), consistently not only with its additional central double bond, but also because in P2 the two phenyl rings are significantly tilted as a consequence of the steric hindrance between $\mathrm{H}$ atoms in ortho position. Instead gap differences between TrPE and $\mathrm{P}_{3}$ and especially between TPE and $\mathrm{P}_{4}$ are much smaller, consistently with TrPE and TPE having one or two rings not complanar ${ }^{6}$ with the main DPE conjugated system (which appears to have an effective conjugation comparable to $\mathrm{P}_{4}$ ).

Turning to the thienyl series, again the DTE gap is narrower than the T2 one, but the difference is much smaller; as in linear $\alpha$ oligothiophenes the steric ortho effect should not be considered. Analogously to the phenyl series, gap differences between TrTE and $T_{3}$ and between TTE and $T_{4}$ are much smaller than that between DTE and $T_{2}$, consistently with lack of complanarity of one or two rings. ${ }^{6}$

The solvent effect on the peak potentials (normalized against the ferrocene couple to enable correct comparison of the solvent effect) is significant but not remarkable. The first reduction peak potentials are, for all available comparison cases, invariably more positive (easier reduction) in $\mathrm{ACN}$, while first oxidation ones are less positive (easier oxidation) in $\mathrm{ACN}$ for thienylethylenes, but in DCM (see SI) for the phenyl and furyl ethylene series.

\section{Electrooligomerization}

CV experiments also included a study of the oligomerization ability of the nine monomers by electrooxidation and subsequent radical cation coupling, according to the classical mechanism described by Heinze. ${ }^{16}$ Although the electrooligomerization of some monomers had already been reported in the literature (e.g. the many works on $\mathrm{DTE}^{16}$ and $\mathrm{DFE}^{17}$ ), the present investigation was carried out on the whole molecule group since reliable comparison can only be achieved at constant working protocol (solvent, supporting electrolyte, monomer concentration, potential scan rate, electrode surface etc.). In particular, repeated potential cycles (in most cases 36 ) were performed around the first oxidation peak of each monomer in the cases where electrodeposition was achieved. The stability of the oligomer film was checked performing further oxidative cycles in monomer free solution, at first at the same scan rate as the electrodeposition step, then at higher or lower rates, to evaluate the facility of the doping/undoping processes. Finally, widening the potential cycle towards negative potentials afforded evaluation of the oligomer film reducibility and of possible charge trapping effects. A selection of results is provided in SI (Figures EC 6-11); values of maxima potentials for first oxidation and, when available, reduction are collected in Table 3.

Phenyl derivatives. As above mentioned, the CV patterns of the monomers of this family show increasing chemical reversibility with increasing scan rate (increasing symmetrical return peak ant constant scan rate), pointing to the radical anions and radical cations formed in the first oxidation and first oxidation processes being rather stable. Consistently with this observation, almost no electrodeposition was achieved performing oxidative cycles with these monomers in our working conditions.

Thienyl derivatives. The monomers of this family, which display chemically irreversible oxidation peaks, give electroactive films of much higher effective conjugation than the starting monomers (as evident from their oxidation and reduction potentials reported in Table 2).

Table 3. Oxidation potentials of the monomers and of their oligomer films electrochemically grown on GC as working electrode, in DCM and ACN

\begin{tabular}{|c|c|c|c|c|c|}
\hline \multirow[t]{2}{*}{ Compd. } & & \multicolumn{2}{|c|}{$\begin{array}{c}\text { Anodic peak } \\
(\mathrm{E} / \mathrm{V} \text { vs } \mathrm{Fc}+\mid \mathrm{Fc})\end{array}$} & \multicolumn{2}{|c|}{$\begin{array}{c}\text { Cathodic peak } \\
\text { (E /V vs Fc+|Fc) }\end{array}$} \\
\hline & & DCM & ACN & DCM & $\mathrm{ACN}$ \\
\hline \multirow[t]{2}{*}{ DTE } & Mon. & 0.75 & 0.72 & 1 & -2.54 \\
\hline & Film & 0.30 & 0.67 & -2.24 & I \\
\hline \multirow{2}{*}{ TrTE } & Mon. & 0.79 & 0.72 & 1 & -2.44 \\
\hline & Film & 0.43 & 0.52 & -1.86 & -1.95 \\
\hline \multirow[t]{2}{*}{ TTE } & Mon. & 0.54 & $0.54,0.64$ & -2.34 & -2.12 \\
\hline & Film & 0.38 & 1 & 1 & 1 \\
\hline \multirow[t]{2}{*}{ DFE } & Mon. & 0.63 & 0.71 & 1 & -2.72 \\
\hline & Film & 0.20 & 0.37 & 1 & 1 \\
\hline \multirow[t]{2}{*}{ TrFE } & Mon. & 0.59 & 0.61 & 1 & -2.49 \\
\hline & Film & 0.40 & 1 & 1 & 1 \\
\hline \multirow[t]{2}{*}{ TFE } & Mon. & 0.47 & 0.57 & -2.4 & -2.19 \\
\hline & Film & 0.44 & 1 & / & 1 \\
\hline
\end{tabular}

The electrooligomerization ability however remarkably decreases with increasing ring number, and is very slow but still perceivable for TTE. This trend might be a consequence of either increasing stabilization of the radical cation and/or increasing sterical hindrance due to the higher number of thiophenes (actually, the TTE monomer oxidation peak shows some return at $2 \mathrm{~V} / \mathrm{s}$ scan rate, Flgure EC4 in the SI). In DCM the oligomerization process is fast for DTE, slow for TrTE. In ACN the process is fast for DTE (even faster than in DCM), slow for TrTE (like in DCM), negligible for TTE.

In the DTE case, the first oxidation peak of the monomer is preceded by a prepeak (well evidenced in Figure EC4); cycling around the latter only, without including the main one, results in oligomerization of a film with similar patterns, but at a much slower rate (Figure EC6.a in the SI). Performing stability cycles on the resulting oligo-DTE films, maxima appear significantly shifting to more extreme potentials with increasing scan rate, pointing to the doping/undoping process being kinetically hindered (Figure EC6.b). This effect is particularly evident working in ACN on GC, as well as working on ITO transparent electrodes, which also affords observation of the oligomer film electrochromism.

With TrTE, the electrodeposition is slower working in the same conditions, as already mentioned (but can be greatly increased increasing the monomer concentration, Figure EC7a), and proceeds at a similar rate in the two solvents. Stability cycles are much more symmetrical than in the DTE case, with maxima 
nearly constant with scan rate, pointing to a facile doping/undoping process. This could be linked to the slower, more regular electrodeposition and to the lower thickness of the deposited film, since it does not apply to the films deposited at higher monomer concentration on DCM Very thin films are electrodeposited from TTE in DCM (Figure EC8) but not in ACN. Their stability cycles appear rather symmetrical and unaffected by scan rate, as in the TrTE case and possibly for the same reasons. Widening the potential range to study first reduction processes, the films appear easier reduced than the monomers, and able to release the negative charge at potentials only slightly more positive than the reduction peak ones. Very slight charge trapping effects could be observed in a few cases, actually those of the thicker films, i.e. oligo-DTE grown in ACN and DCM and oligo-TrTE grown in DCM with a tenfold monomer concentration.

Furyl derivatives. Even the furyl derivative family gives electrooligomerization, but with comparatively slower rates than the thienyl one. Also in this series the same trend of decreasing oligomerization ability with increasing number of heteroaromatic rings is observed. DFE displays a slower, more regular electrooligomerization than DTE (its behaviour looking similar to that of TrTE). The stability cycles of the film grown on GC in $\mathrm{ACN}$ are particularly symmetrical (like oligomerization cycles, too) and constant with scan rate, pointing to a facile doping/undoping process. TrFE oligomerization is slower than the DFE one, and apparently the film lacks stability upon performing the stability cycles. TFE oligomerization is even slower, and, again, the resulting films appear unstable upon cycling.

\section{Electrochemical impedance spectroscopy (EIS) experiments.}

Films obtained by electrooligomerization were also studied in monomer-free solution by comparing their electrochemical impedance spectra at different potentials, corresponding to increasingly positive film charging. Details are reported in the SI, with Nyquist, Bode modulus, and Bode phase diagrams for selected cases (Figures EC12-18). Evaluating them qualitatively according to the equivalent circuit proposed by Musiani ${ }^{18}$ the presence of a charge transfer resistance $R_{\mathrm{CT}}$ appears nearly unperceivable and rapidly disappearing with increasing potential (i.e. with increasing degree of positive film charging), when not unperceivable at all potentials; this confirms a facile oxidative charge transfer to the film. Some slight differences can however be noticed between different films; in particular, the $R_{\mathrm{CT}}$ presence appears more evident (a) with increasing film thickness, (b) growing the film on ITO rather than GC; (c) after performing stability cycles; (d) for a TrTE film respect to a TTE one (both of them grown with the same protocol on the same kind of support).

\section{Optical properties}

The absorption and emission data of the whole ethylene series, are collected in Table 3, in which we present the photophysical data of DFE, TrFE, TFE, TrTE and TrPE molecules, objects of the present paper, and those of DPE, TPE, DTE and TTE reported in our previous paper. ${ }^{6}$

The absorption spectra of the DFE, TrFE, TFE, TrTE and TrPE, recorded in dichloromethane (DCM) solution, are shown in figure 4.

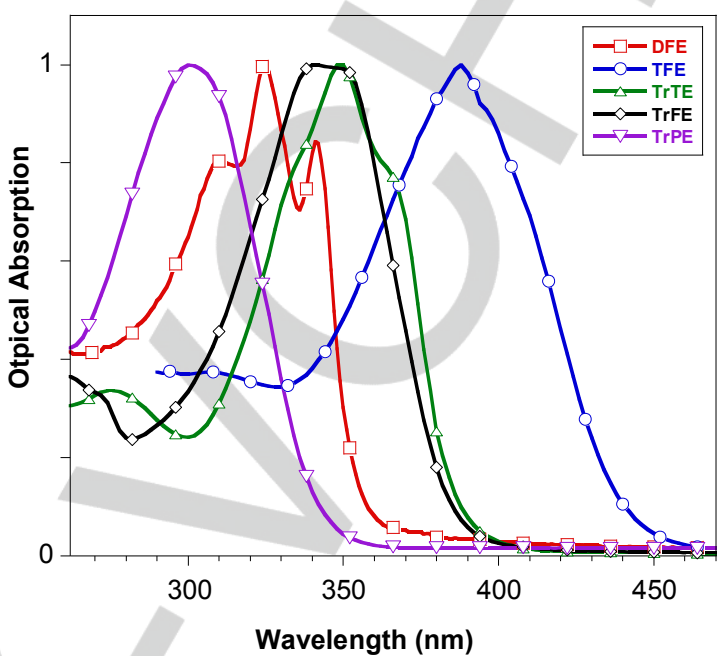

Figure 4. Normalized optical absorption spectra of DFE, TFE, TrTE, TrFE, and $\operatorname{TrPE}$ in $10^{-5} \mathrm{M}$ DCM solution

The absorption of the furan containing ethylenes shows a redshift from 324 to $388 \mathrm{~nm}$ with increasing the number of furanyl units (see Table 4). DFE molecule shows a well resolved vibronic structure with peaks at 310,324 and $341 \mathrm{~nm}$. When three (TrFE) and four furanyl groups (TFE) are present, the absorption band broadens losing the vibronic structure and redshifts of 0.19 and $0.63 \mathrm{eV}$, respectively, compared to the molecule with only two furanyl groups (DFE). The red-shift of the absorption from DFE to TFE is in agreement with the reduction of the energy gap of about $0.6-0.7 \mathrm{eV}$ obtained from the $\mathrm{CV}$ results (see Table 1).

\begin{tabular}{cccc}
\multicolumn{4}{c}{ Table 4. Optical properties of (hetero)arylethylene series } \\
\hline Compd. & $\lambda_{\text {abs }}{ }^{\left[{ }^{[a]}\right.}[\mathrm{nm}]$ & $\lambda e \mathrm{~m}^{[\mathrm{bb}]}[\mathrm{nm}]$ & $\mathrm{QY}^{[\mathrm{bb}]}$ \\
\hline DPE & 300 & 385 & 0.70 \\
TrPE & 300 & 430 & 0.26 \\
TPE & 307 & 445 & 0.37 \\
DTE & 343 & 438 & 0.30 \\
TrTE & 350 & 480 & 0.03 \\
TTE & 370 & 420 & 0.06 \\
DFE & 324 & 388 & 0.33 \\
TrFE & 341 & 435 & $<0.01$ \\
TFE & 388 & 495 & 0.17
\end{tabular}

[a] absorption peak position in DCM solutions $\left(10^{-5} \mathrm{M}\right)$. [b] emission peak position and PL QY in the solid state at room temperature. 
Comparing the absorptions of trisubstituted derivatives (TrPE, TrTE and TrFE) we observe that TrPE and TrFE displays broad absorption bands centered at about 300 and $341 \mathrm{~nm}$, while TrTE shows a partially resolved vibronic structure with a maximum at 350 and a shoulder at $368 \mathrm{~nm}$.

Most of these molecules, similarly to TPE derivatives, do not show any detectable emission in solution but shows a quite intense emission in the solid state, therefore displaying an aggregation-induced emission behavior. ${ }^{19}$

The photoluminescence (PL) spectra of DFE, TrFE, TFE, TrTE and TrPE in the solid state at room temperature are reported in figure 5 . The emission goes from a deep blue for the smaller molecule, DFE, to a green emission for the larger one, TFE.

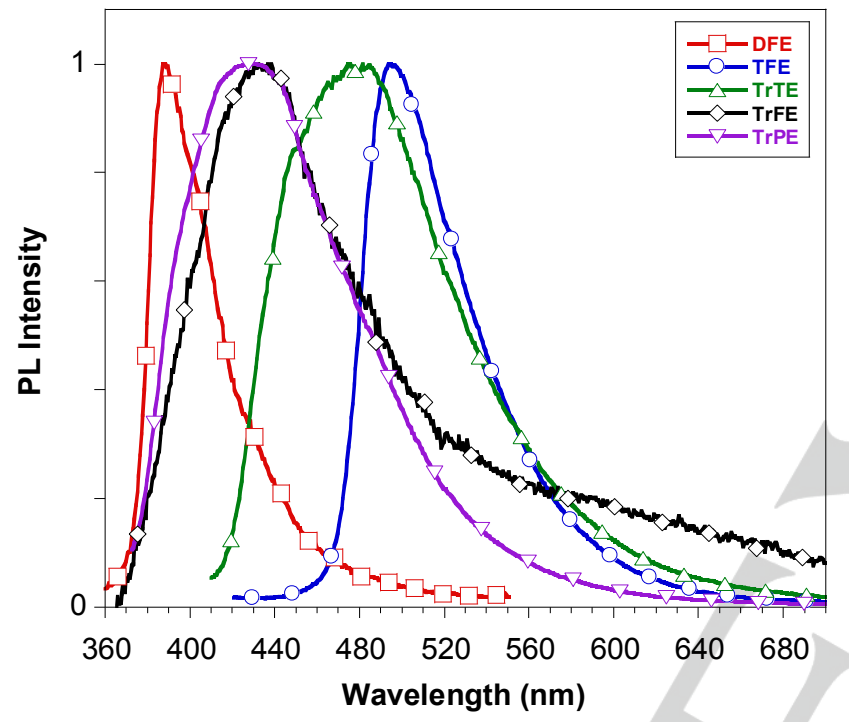

Figure 5. Normalized PL spectra of DFE, TrFE, TFE, TrTE and TrPE, in the solid state.

The PL spectrum of DFE is characterized by a narrow band peaked at $388 \mathrm{~nm}$ and a PL Quantum Yield (QY) of 0.33. The TrFE molecule shows a broad emission band, centered at about $435 \mathrm{~nm}$ and a very low PL QY. The TFE molecule is characterized by a red-shifted PL band with a maximum at 495 $\mathrm{nm}$ and $\mathrm{QY}$ of 0.17 .

A bathochromic shift of $107 \mathrm{~nm}(0.69 \mathrm{eV})$ in the emission band is observed with four furanyl substituents (TFE), when compared to the emission band of DFE, mirroring the behavior illustrated in the absorption spectra of the solutions (see Table 4).

The new thiophene containing TrTE molecule is characterized by a broad emission band, with a peak at $480 \mathrm{~nm}$, and a PL QY of 0.03 , while the corresponding phenyl substituted TrPE molecule shows a broad emission band with a position similar to that of TrFE but a higher PL QY of 0.26 .

We noted that the compounds with three aromatic groups display the lowest emission efficiency in all the series of ethylenes.

Due to the propeller-shape structure of this class of molecules, the related AIE mechanism could be due to the restriction of intramolecular rotation RIR mechanism ${ }^{3}$ and basing on this we might assume that the compounds with three aromatic groups have lower tendency to aggregate in rigid structures. This is evident in the case of the TrFE molecule that appears in the form of oil, thus its emission properties are expected to be similar to those of the corresponding solution.

If molecules of the whole series are compared, we note that the highest emissive are those with the phenyl ring substituents, while the lowest ones are those with the thiophene ring, in agreement with the different emission efficiency of these systems. ${ }^{20}$

\section{Computational studies of TPE, TTE and TFE}

Ab-initio computations have been performed on tetra-substituted subset of the above reported compounds namely TPE, TTE and TFE.

Hartree-Fock and DFT computations have been done using GAUSSIAN $09^{21}$ suite of programs. Meta-hybrid GGA functional M06 ${ }^{22}$ has been chosen which accounts for no covalent interactions and gives energy barriers enough accurate. In HF computations valence double zeta plus polarization on all atoms basis set, $6-31 \mathrm{~g}(\mathrm{~d}, \mathrm{p}){ }^{23}$ has been used. In DFT valence triple zeta plus polarization on all atoms plus diffused on heavy atoms basis set, $6-311+g(d, p)$, has been used. ${ }^{24}$

Each structure has been fully optimized and vibrational frequencies have been computed to confirm the stationary point type.

From the data described below that have been obtained by using both Hartree-Fock (HF) and density functional theory (DFT), it has emerged that both TTE and TFE can exist in several conformers and they differ from one another by few $\mathrm{Kcal} / \mathrm{mol}$, so that all of them are accessible from the energetic point of view.

Both tetra(2-furyl)ethylene (TFE) and tetra(2-thienyl)ethene (TTE) have rings tilted out of plane as it happens in tetraphenylethylene (TPE) because of the steric hindrance of the rings. The ethylene bond, also, is rather twisted featuring an angle between $10^{\circ}$ and $20^{\circ}$. The relevant geometrical data are reported in Table 5.

Seven distinct structures have been identified for both TTE and TFE. They are hereafter indicated by the form $c+t-c+t-$, where $c$ and $t$ stand for cis and trans and indicate the orientation of the heteroatom with respect to the ethylene double bond, and + and - indicate the relative position of the heteroatom below or over the mean plane of the molecule. The order is indicated by the subscript of torsion angles in the following figure 6 .

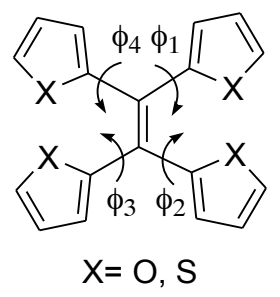

Figure 6. Torsion angles in tetrasubstituted ethylene structure 


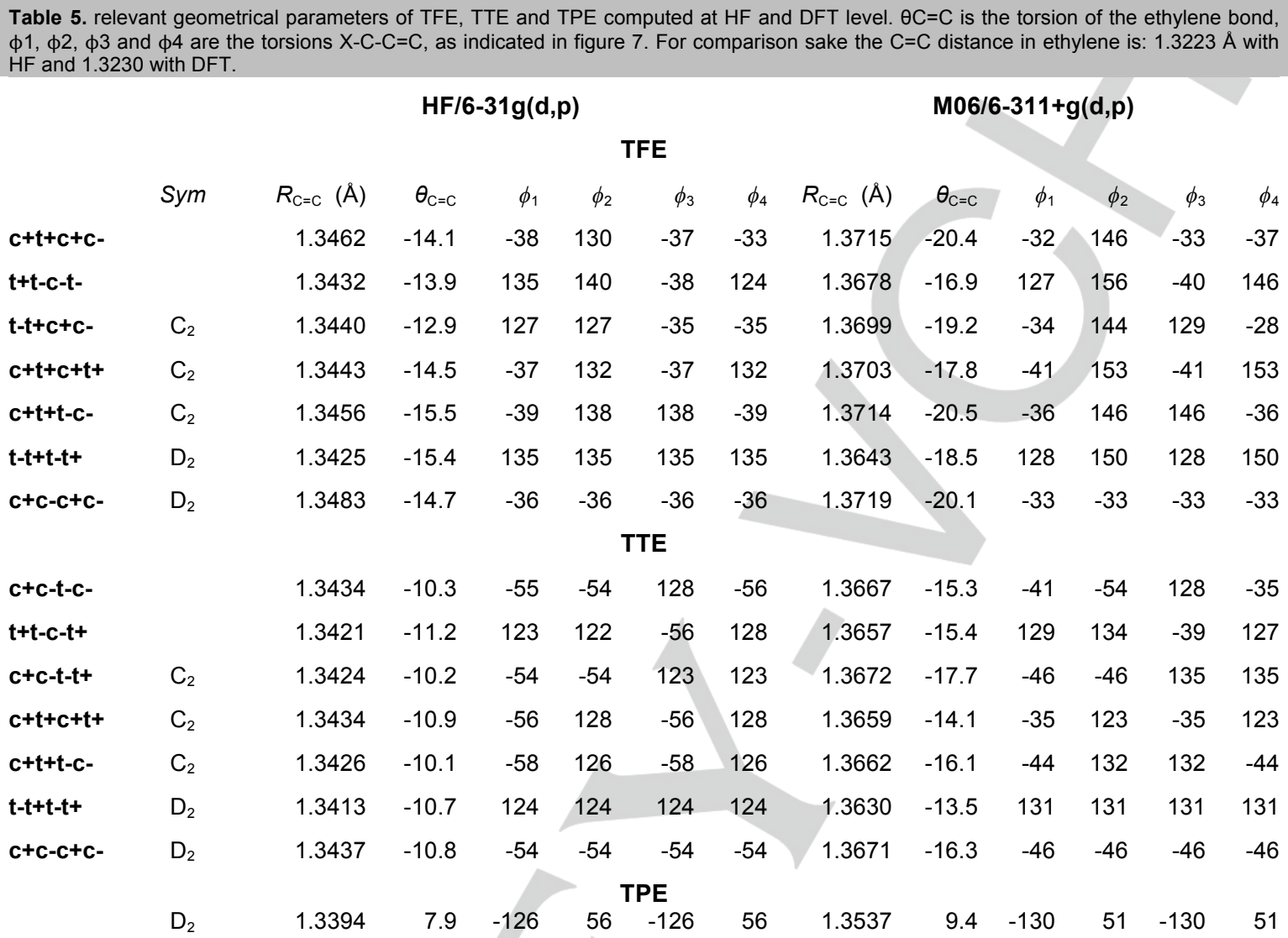

The structures of TTE, TFE and TPE have always the rings strongly twisted respect the $\mathrm{C}=\mathrm{C}$ double bond plane.

The only experimental structure found in Cambridge Structural Database ${ }^{25}$ shows that in the DTE molecule the two tiophene rings are coplanar, but a computation on isolated molecule at experimental structure gave a high order saddle-point with imaginary normal coordinates showing rotations of these rings. Therefore the coplanar configuration is to be attributed to packing interactions.

As can be deduced from table 5 the plane of the ethylene moiety is more twisted in TFE than in TTE with a consequent elongation of the $\mathrm{C}=\mathrm{C}$ distance while TPE shows only a slight torsion. As a consequence of the reduced torsion, in TTE the thiophene rings are more out of plane.

The M06 results are in agreement with the HF ones; as this DFT functional contains a certain degree of correlation error correction it gives a $\mathrm{C}=\mathrm{C}$ distance longer than those computed with HF: therefore there is a reduction of hindrance which produces a lesser twisting of both the $\mathrm{C}=\mathrm{C}$ bond and the rings. Energies of different conformers are reported in Table 6 and in any case all the conformers lay in a range of maximum 3 $\mathrm{kcal} / \mathrm{mol}$. A complete analysis of the reaction pathways among such a number of conformers is outside the aim of this work.
Relaxed scans, with both methods, of the complete rotation of a single ring either in TFE or in TTE showed barriers of few $\mathrm{kcal} / \mathrm{mol}$ and anyway ranging (M06/6-311+g(d,p)) from 3 to 5 $\mathrm{kcal} / \mathrm{mol}$ for TFE, from 0.5 to $2 \mathrm{kcal} / \mathrm{mol}$ for TTE and from 5 to 7 $\mathrm{kcal} / \mathrm{mol}$ for TPE. As a consequence, in experimental condition, all the conformers should be present with a rather uniform distribution both in gas and diluted solution phases. This means that experimental measures are a thermodynamic mean on all configurations.

In Table 7 are reported the gaps between the highest occupied (HOMO) and lowest unoccupied (LUMO) orbitals. It should be noted that HF eigenvalues are true energies of the orbitals and, in the scheme of Koopmans theorem, the eigenvalue of HOMO is the opposite of the energy to extract an electron and that of LUMO is the energy to add an electron, so the difference between LUMO and HOMO is an adiabatic approximation of excitation energy to the first excited electronic state. Otherwise the DFT eigenvalues are the derivate of energy with respect to the occupation number of pseudo- orbitals: in fact in Kohn-Sham method the "orbitals" are not true wave functions but only a tool to get the correct electron density.

With a heavy approximation we can obtain the energy, $\Delta \mathrm{E}$ to extract an electron from i-th pseudo-orbital: 


$$
\Delta E=-\int_{2}^{1} \frac{\partial E(n)}{\partial n_{i}} d n_{i}
$$

If we assume that $\left(\partial E(n) / \partial n_{i}\right.$ is constant from $n_{i}=2$ to $n_{i}=1$, which is a rough approximation then: $\Delta E=-\partial E(n) / \partial n_{i}=-\varepsilon_{i}{ }^{(D F T)}$

Anyway it is known that LUMO energies, as computed by DFT methods, ${ }^{26}$ are generally unrealistically low, so, while HF gaps are expected to be too high, the DFT gap are expected to be too low.

In fact the differences between relaxed computations for cation and anion give values more in line with HF computed HOMOLUMO gaps: TFE $\mathrm{t}-\mathrm{t}+\mathrm{t}-\mathrm{t}+7.51$, TFE $\mathrm{c}+\mathrm{c}-\mathrm{c}+\mathrm{c}-7.39$, TTE $\mathrm{t}-\mathrm{t}+\mathrm{t}-\mathrm{t}+$ 7.62, TTE $\mathrm{c}+\mathrm{c}-\mathrm{C}+\mathrm{c}-$ 7.58. In table 6 are also reported the energies of the first electronic transition as computed with the time dependent DFT model (TD-DFT). These values are in line with the $\mathrm{CV}$ measures

Table 6. Energies and relative energies of TFE and TTE

\begin{tabular}{|c|c|c|c|c|}
\hline \multirow{4}{*}{ 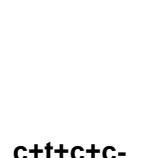 } & \multirow{2}{*}{$\begin{array}{c}\text { HF/6-31g(d,p) } \\
E_{\text {tot }}(\text { a.u. })^{[a]}\end{array}$} & \multicolumn{3}{|c|}{$M 06 / 6-311+g(d, p)$} \\
\hline & & $\Delta \mathrm{E}^{[\mathrm{b}]}$ & $\mathrm{E}_{\text {tot }}(\mathrm{a} . \mathrm{u} .)^{[\mathrm{a}]}$ & $\Delta \mathrm{E}^{[\mathrm{b}]}$ \\
\hline & \multicolumn{4}{|c|}{ TFE } \\
\hline & -987.679483 & 0.80 & -993.259444 & 0.82 \\
\hline$t+t-c-t-$ & -987.678242 & 1.57 & -993.257334 & 2.15 \\
\hline$t-t+c+c-$ & -987.679091 & 1.04 & -993.257772 & 1.87 \\
\hline$c+t+c+t+$ & -987.678133 & 1.64 & -993.258392 & 1.48 \\
\hline$c+t+t-c-$ & -987.678748 & 1.26 & -993.258728 & 1.27 \\
\hline$t-t+t-t+$ & -987.677909 & 1.78 & -993.255831 & 3.09 \\
\hline \multirow[t]{2}{*}{$c+c-c+c+$} & -987.680750 & 0.00 & -993.260758 & 0.00 \\
\hline & \multicolumn{4}{|c|}{ TTE } \\
\hline$c+t+c+c-$ & -2278.334534 & 1.97 & -228 & 0.31 \\
\hline$t+t-c-t-$ & -2278.336528 & 0.72 & -2285.200234 & 0.33 \\
\hline$t-t+c+c-$ & -2278.335672 & 1.26 & -2285.200031 & 0.46 \\
\hline$c+t+c+t+$ & -2278.335277 & 1.50 & -2285.200711 & 0.03 \\
\hline$c+t+t-c-$ & -2278.335450 & 1.40 & -2285.200757 & 0.00 \\
\hline$t-t+t-t+$ & -2278.337675 & 0.00 & -2285.199805 & 0.60 \\
\hline$c+c-c+c+$ & -2278.333693 & 2.50 & -2285.200020 & 0.46 \\
\hline
\end{tabular}

[a] $T_{\text {ot }}$ is the sum of the electronic energy and the zero point vibrational energy computed in harmonic approximation. [b] relative energy in $\mathrm{Kcal} / \mathrm{mol}$..

As can be expected, in the presence of the above mentioned strong torsions of the $\mathrm{C}-\mathrm{C}$ bonds between ethylene and rings the HOMO orbitals, as shown in Figure 7 , do not have any hint of conjugation between rings and $\mathrm{C}=\mathrm{C}$ ethylene bond.

Figure 8 shows that the HOMO-LUMO gap decreases when the $\mathrm{C}=\mathrm{C}$ bond length increases, or better it is almost linear with $1 /(R C=C) 2$ as for the "particle in a box". This fact is explained by inspecting the shape of the HOMO orbitals (Figure 7) which are mainly a $\pi$ bonding orbital of the ethylene moiety and the LUMO is the corresponding anti-bonding one.
Table 7. HOMO and LUMO orbital energies of TFE, TTE and TPE along with HOMO-LUMO gap, $\Delta \varepsilon$. The LUMO HOMO gap in ethylene is $15.01 \mathrm{eV}(\mathrm{HF})$ and $7.76 \mathrm{eV}$ (DFT). TDDFT are the energies of the first electronic transition computed by time dependent $M 06 / 6-311+g(d, p)$. For comparison sake, in ethylene the LUMO HOMO gap is $15.01 \mathrm{eV}$ (HF) and $7.76 \mathrm{eV}$ (DFT), the first electronic transition is at $6.67 \mathrm{eV}$

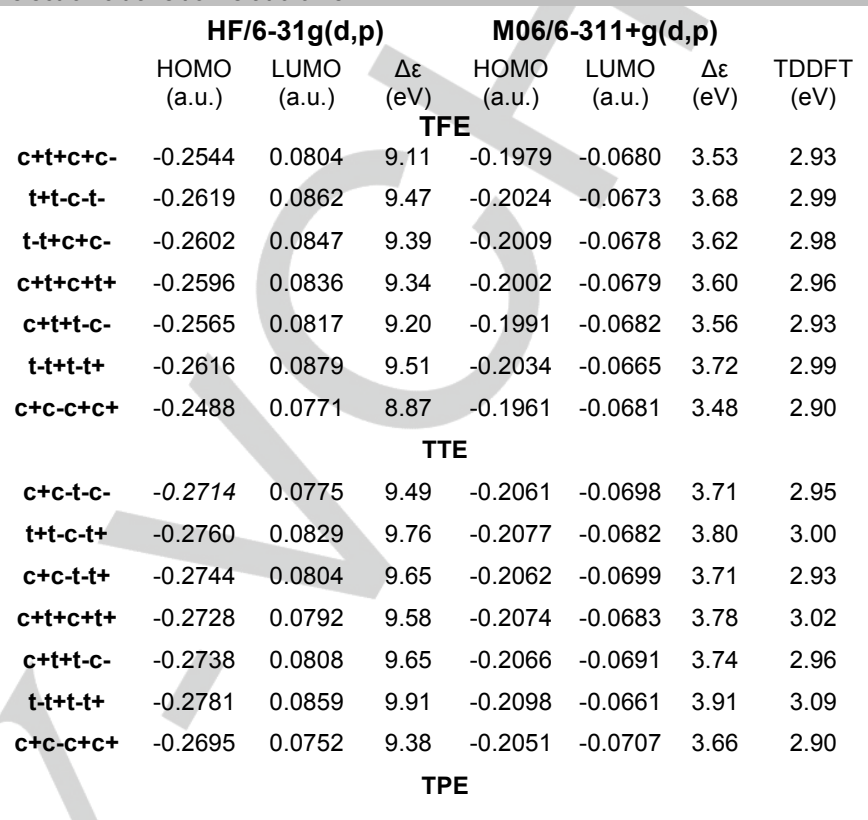

$\begin{array}{lllllll}0.2728 & 0.1017 & 10.19 & -0.2192 & -0.0518 & 4.56 & 10.19\end{array}$
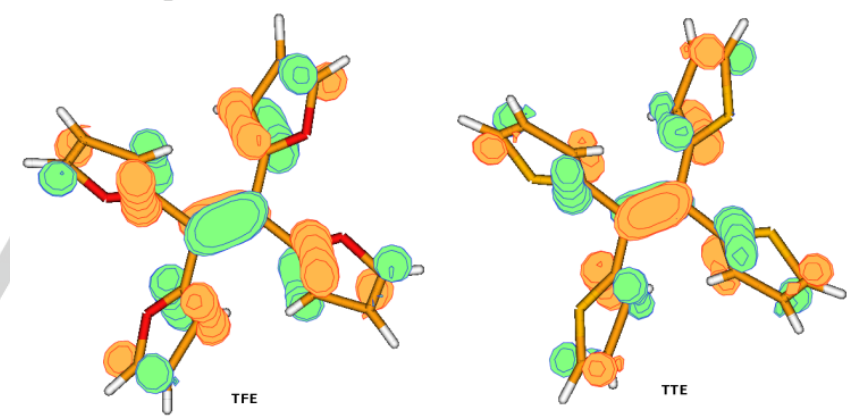

Figure 7. Homo of TFE and TTE computed at HF levels.
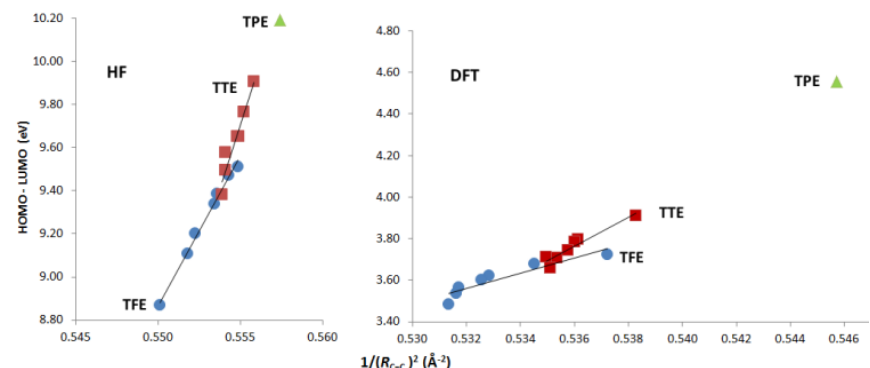

Figure 8. HOMO-LUMO energy differences as function of $\mathrm{C}=\mathrm{C}$ bond distance in the ethylene moiety, computed with $\mathrm{HF} / 6-31 \mathrm{~g}(\mathrm{~d}, \mathrm{p})$ on left and M06/6$311+g(d, p)$ on right. 
The dependency of HOMO-LUMO energy gap on $\mathrm{C}=\mathrm{C}$ bond distance is neater in HF results because the above pointed to difference between HF and DFT orbitals. Figure 8 also shows a difference between TFE and TTE: TTE gaps have a steeper slope and correlate well with TPE gap.

This difference is paralleled by $\mathrm{APT}^{27}$ (Atomic Polarization Charge) values, whatever the model: in TFE the oxygen is always negative, with a greater charge when it is in cis, while in TTE sulfur atom is always slightly charged generally positively by values not far from TPE with a slightly greater charge when the sulfur is in cis configuration.

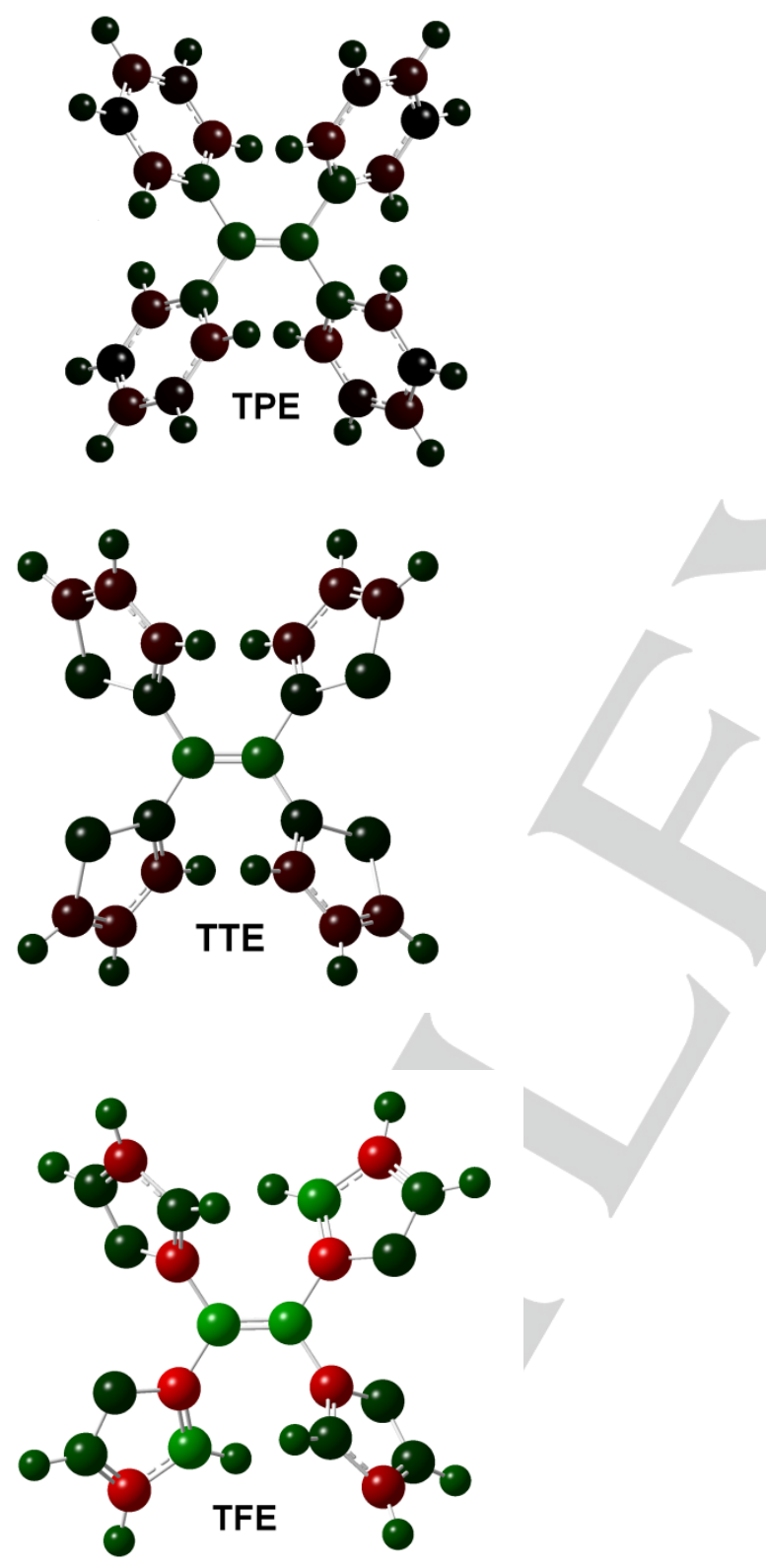

Figure 9. Atomic APT charges in colours (red is positive, green is negative) as computed by the DFT method. From left TPE, TTE and TFE
So the HOMO-LUMO gap in such type of molecules depends mainly by the stretching effect of substituents on the $\mathrm{C}=\mathrm{C}$ bond length and on the presence of strongly negative charged groups in the substituents. Above reported results are for the insulated molecule, if the packing with other molecules increases the hindrance effect we shall expect a reduction of the gap.

The APT atomic charge distribution are showed in colors in Figure 9, (the numerical values can be found in SI). It can be noted that the most relevant feature, going from TPE to TTE and then to TFE, is the increasing of the number and the values of negative APT charges in the rings. The trend is confirmed by a number of integrations of AIM charges. ${ }^{28}$

Therefore we can conclude that molecular orbitals involved in the electronic transition are mainly $\pi$ orbitals of ethylene group but the gap is electrostatically modified by the charges on the substituents: an increase in negative values leads to a lower HOMO-LUMO gap and this is connected to the different slope of the dependence of the gap on the ethylene bond length.

\section{Conclusions}

A detailed photophysical and electrochemical comparative study has been achieved for three parallel series of phenyl, thienyl and furyl substituted ethylenes, supported by the computational study of selected terms. A systematic, exhaustive electrochemical investigation by cyclic voltammetry, also supported by electrochemical impedance spectroscopy, enabled to study electronic properties and oligomerization ability of the (hetero)aryl ethylene monomers as a function of the number and type of aromatic rings. The central double bond significantly contributes to the overall $\pi$-conjugation efficiency, on which the presence of tilted aromatic rings plays a key role. With respect to phenyl-substituted ethylenes, the energy gaps of thiophene- and furan-based ones are comparatively smaller and displaced to higher energies, consistently with higher electron-rich character. Unlike phenyl-based systems, both heterocycle families give electrooligomerization, at a rate comparatively faster for the thienyl series, and decreasing for both series with increasing number of heteroaromatic rings. The electrodeposited oligomer films show facile electron transfer as well as electrochromic effects.

Results from the photophysical study confirm the above considerations, indicating a remarkable red shift for the heteroaryl systems compared to the phenyl series. At the same time, in all of the cases a red shift is observed by increasing the number of rings on the double bond. The highest emission efficiency was observed for phenyl-substituted ethylenes series, while a lower one was displayed by trisubstituted ethylenes TrPE, TrTE and TrFE, when compared with di-and tetrasubstituted series, possibly indicating to their lower ability to aggregate in rigid structures.

Computational studies (HF and DFT) performed on TTE, TFE and TPE, indicate that the three molecules exist in seven distinct conformers with an energy barrier as low as $3 \mathrm{kcal} / \mathrm{mol}$. 
Moreover, the four (hetero)aryl rings are strongly twisted with respect to the carbon-carbon double bond plane: therefore, the partial planarity of these systems mentioned in the literature could be attributed to packing interactions in the solid state. The same trend observed from electrochemical study is found in calculated energy gaps that is lower gaps are found for TTE and TFE with respect to TPE.

A palette of interpretative/predictive guidelines is thus made available concerning the redox and spectroscopic properties of (hetero)arylethylenes as a function of their molecular structure, which could be particularly useful in view of their possible application, as monomers and/or related oligomer films, in various molecular devices.

\section{Experimental Section}

\section{General information}

Unless otherwise specified, all the reactions were performed under nitrogen atmosphere using standard techniques using flask equipped with a magnetic stirring bar, septum inlet, and reflux condenser when necessary. All reagents and solvents were obtained from highest grade commercial sources and used without further purification. Anhydrous solvents were purged with nitrogen before use. The reaction outcome was monitored by TLC silica gel plates and HPLC. All chromatographic separations were carried out on Merck silica gel (60 $\mu, 230-400$ mesh). Melting points were obtained with a Büchi B-540 melting point apparatus and are uncorrected. ${ }^{1} \mathrm{H}$ NMR and ${ }^{13} \mathrm{C}$ NMR spectra were recorded on a Bruker AVANCE DRX-400, Bruker AC300 and AMX $300 \mathrm{MHz}$ spectrometers; the chemical shifts $(\delta)$ are reported in parts per million relative to the solvent residual peak $\left(\mathrm{CDCl}_{3}\right)$. HPLC analyses were performed on an Agilent 1100 series equipped with a PDA detector and the reverse phase ZORBAX Eclipse XBD-C18, $(4.6 \times 150 \mathrm{~mm}, 5 \mu \mathrm{m}$ particle size) eluent: $\mathrm{CH}_{3} \mathrm{CN} / \mathrm{H}_{2} \mathrm{O}$, (1 $\left.\mathrm{mL} / \mathrm{min}\right)$. DPE, TPE, TrPE, DTE and dioxaborolane $\mathbf{6}$ and $\mathbf{7}$ are commercially available compounds (SigmaAldrich).

Electrochemistry: The cyclovoltammetric study of all the compounds was performed at scan rates typically ranging $0.05-2 \mathrm{~V} s-1$. The concentration of substrates was tipically $0.0005 \mathrm{M}$ in $\mathrm{CH} 2 \mathrm{Cl} 2$ and $\mathrm{CH} 3 \mathrm{CN}$ with tetrabutyl ammonium hexafluorophosphate $\left(\mathrm{TBAPF}_{6}\right) 0.1 \mathrm{M}$ as the supporting electrolyte. Glassy carbon (GC) electrode for was used $\mathrm{CV}$ experiments, electrodeposition and characterization of oligomer films were conducted on both on GC and on ITO (indium tin oxide) transparent electrode. The solutions were deaerated by $\mathrm{N}_{2}$ bubbling. The ohmic drop has been compensated by the positive feedback technique ${ }^{29}$ Two halfcycles were run: one anodic and one cathodic, started at a potential where no electrode processes take place, in order to avoid modification of the signals of interest as a consequence of the presence of products of the opposite reaction. The experiments were carried out using an AUTOLAB PGSTAT potentiostat (EcoChemie, The Netherlands) run by a PC with GPES software. The working electrode was a glassy carbon GC one $(A M E L$, diameter $=1.5 \mathrm{~mm}$ ) cleaned by diamond powder (Aldrich, diameter $=1 \mu \mathrm{m}$ ) on a wet cloth (Struers DP-Nap). The counter electrode was a platinum wire while the reference electrode was an aqueous saturated calomel electrode (SCE), having vs the $\mathrm{Fc}+\mid \mathrm{Fc}$ couple (the intersolvental redox potential reference currently recommended by IUPAC) ${ }^{30}$ a difference of $-0.495 \mathrm{~V}$ in $\mathrm{CH}_{2} \mathrm{Cl}_{2}$ and $-0.391 \mathrm{~V}$ in $\mathrm{CH}_{3} \mathrm{CN}$.

Optical Characterization: Optical absorption measurements were performed using a Perkin-Elmer Lambda-9 spectrometer on dichloromethane diluted solutions (10-5 M). Photoluminescence (PL) spectra were recorded at room temperature by using a 270M SPEX spectrometer equipped with a N2 cooled SPEX Jobin Yvon CCD (charge-coupled device) detector, and by exciting with a Xenon lamp connected to a Jobin Yvon Gemini monochromator for the wavelength selection. The spectra were corrected for the instrument response. DFE, TrFE, TFE, TrTE and TrPE were excited at $365 \mathrm{~nm}, 350 \mathrm{~nm}, 420 \mathrm{~nm}$, $380 \mathrm{~nm}$ and $365 \mathrm{~nm}$, respectively. The PL quantum yields (QY) on solidstate materials were obtained using a homemade integration sphere, following the procedure reported elsewhere, ${ }^{31}$ and a monochromated Xenon lamp. The excitation wavelengths were the same used for recording the $\mathrm{PL}$ spectra.

\section{Synthesis of DFE, TFE, TTE: general procedure}

To a solution of carbonyl compounds 1-3 $(20 \mathrm{mmol})$ in dry THF (50 mL) cooled to $--20^{\circ} \mathrm{C}, \mathrm{TiCl} 4(24 \mathrm{mmol}, 1.2 \mathrm{eq})$ was slowly added. The reaction mixture was stirred $20 \mathrm{~min}$ at $-20^{\circ} \mathrm{C}$, then zinc $(50 \mathrm{mmol}, 2.5 \mathrm{eq})$ was added in three portions. The mixture was stirred for $10 \mathrm{~min}$ at the same temperature and then refluxed for $3 \mathrm{~h}$ (in the case of 2 it was stirred at room temperature). After cooling to room temperature, a saturated $\mathrm{Na}_{2} \mathrm{CO}_{3}$ solution $(20 \mathrm{~mL})$ was added and the solvent evaporated. The residue taken-up with $\mathrm{CH}_{2} \mathrm{Cl}_{2}(50 \mathrm{~mL})$ was filtered over a pad of Celite®. The aqueous phase was extracted with $\mathrm{CH}_{2} \mathrm{Cl}_{2}(4 \times 20 \mathrm{~mL})$ and the combined organic phases were washed with $\mathrm{H}_{2} \mathrm{O}(20 \mathrm{~mL})$, dried over $\mathrm{Na}_{2} \mathrm{SO}_{4}$ and evaporated. The residue was purified by column chromatography (hexane/ $\mathrm{CH}_{2} \mathrm{Cl}_{2}, 9: 1$ ) affording the target compounds: DFE (66\%), TFE (49\%), pale-yellow solid m.p. 163-164 ${ }^{\circ} \mathrm{C}$ (not reported in the lit. ${ }^{11}$ ) and TTE 12 (55\%), whose analytical and spectroscopic data were in agreement with those reported in the literature. ${ }^{9,10,11}$

\section{Synthesis of TrTE and TrFE: general procedure}

To a solution of of 2,2-dibromovinylheterocycle 4 or $5(0.5 \mathrm{mmol})$ and appropriate dioxaborolane 6 or $7(0.8 \mathrm{mmol})$ in THF $(5 \mathrm{~mL}), \mathrm{K}_{2} \mathrm{CO}_{3}(1 \mathrm{M}$ solution, $3 \mathrm{~mL}, 3.0 \mathrm{mmol}), \mathrm{Pd}(\mathrm{OAc})_{2}(0.025 \mathrm{mmol})$, and $\mathrm{PPh}_{3}(0.1 \mathrm{mmol})$ were added. The mixture was refluxed for $8 \mathrm{~h}$, then a second portion of appropriate dioxaborolane $(0.7 \mathrm{mmol})$ was added and the mixture refluxed for $8 \mathrm{~h}$. After cooling to room temperature, $\mathrm{H} 2 \mathrm{O}(20 \mathrm{~mL})$ was added and the mixture taken-up with $\mathrm{CH}_{2} \mathrm{Cl}_{2}(20 \mathrm{~mL})$ was filtered over a pad of Celite ${ }^{\circledR}$. The organic phase was separated and the aqueous phase extracted with $\mathrm{CH}_{2} \mathrm{Cl}_{2}(4 \times 15 \mathrm{~mL})$. The combined organic phases were washed with $\mathrm{H}_{2} \mathrm{O}(20 \mathrm{~mL})$ dried over $\mathrm{Na}_{2} \mathrm{SO}_{4}$ and evaporated. The crude product was purified by column chromatography (hexane) affording the target compound.

TrFE (30\%) light brown oil (easy degradable by air-exposure) ${ }^{1} \mathrm{H}-\mathrm{NMR}$ $\left(300 \mathrm{MHz}, \mathrm{CDCl}_{3}\right): \delta, \mathrm{ppm}=7.60(\mathrm{~s}, 1 \mathrm{H}), 7.51(\mathrm{~s}, 1 \mathrm{H}), 7.42(\mathrm{~s}, 1 \mathrm{H}), 7.09$ $(\mathrm{s}, 1 \mathrm{H}), 6.57-6.61(\mathrm{~m}, 2 \mathrm{H}), 6.48(\mathrm{ps}, 1 \mathrm{H}), 6.42(\mathrm{ps}, 1 \mathrm{H}),, 6.30(\mathrm{~d}, 1 \mathrm{H}, \mathrm{J}=$ 2.9), $6.04(\mathrm{~d}, 1 \mathrm{H}, \mathrm{J}=3.3) .{ }^{13} \mathrm{C}-\mathrm{NMR}\left(75 \mathrm{MHz}, \mathrm{CDCl}_{3}\right): \delta, \mathrm{ppm}=153.87$, 152.16, 149.51, 142.70, 142.63, 142.55, 142.12, 118.20, 111.94, 111.83, 111.19, 110.69, 109.29. MS-EI (m/z) C14H10O3: 226 [M+].

TrTE (70\%) yellow solid, m.p.: $58-60{ }^{\circ} \mathrm{C}{ }^{1} \mathrm{H}-\mathrm{NMR}\left(300 \mathrm{MHz}, \mathrm{CDCl}_{3}\right): \delta$, ppm = $7.54(\mathrm{~d}, 1 \mathrm{H}, \mathrm{J}=5.1 \mathrm{~Hz}), 7.38(\mathrm{~s}, 1 \mathrm{H}), 7.21-7.17(\mathrm{~m}, 2 \mathrm{H}), 7.14(\mathrm{~d}$, $1 \mathrm{H}, \mathrm{J}=5.1 \mathrm{~Hz}), 7.08(\mathrm{~d}, 1 \mathrm{H}, \mathrm{J}=3.4 \mathrm{~Hz}), 7.01(\mathrm{~d}, 1 \mathrm{H}, \mathrm{J}=3.5 \mathrm{~Hz}), 6.97-$ $6.90(\mathrm{~m}, 2 \mathrm{H}), 6.83(\mathrm{~d}, 1 \mathrm{H}, \mathrm{J}=3.6 \mathrm{~Hz}) .{ }^{13} \mathrm{C}-\mathrm{NMR}(75 \mathrm{MHz}, \mathrm{CDCl} 3): \delta$, $\mathrm{ppm}=122.98,124.86,125.89,126.42,127.63,127.75,127.81,129.01$, 129.70, 140.32. HRMS-El (m/z): calc. for $\mathrm{C}_{14} \mathrm{H}_{10} \mathrm{~S}_{3}: 273.99446$, found 273.993960 .

Cross McMurry reaction to obtain DFE, TrFE, TFE 
To a solution of carbonyl compounds $1(5 \mathrm{mmol})$ and $2(5 \mathrm{mmol})$ in dry THF ( $30 \mathrm{~mL}$ ) cooled to $-20^{\circ} \mathrm{C}, \mathrm{TiCl}_{4}(24 \mathrm{mmol}, 1.2 \mathrm{eq})$ was slowly added. The reaction mixture was stirred $20 \mathrm{~min}$ at $-20{ }^{\circ} \mathrm{C}$, then zinc $(50 \mathrm{mmol}$, 2.5 eq) was added in three portions. The mixture was stirred for $10 \mathrm{~min}$ at the same temperature, then refluxed for $3 \mathrm{~h}$, (following the disappearance of the starting compounds by TLC: hexane/AcOEt, 9:1). A saturated $\mathrm{Na}_{2} \mathrm{CO}_{3}$ solution was added and the solvent evaporated, then $\mathrm{CH}_{2} \mathrm{Cl}_{2}(30 \mathrm{~mL})$ was added and the mixture was filtered over a pad of Celite $\circledast$. The aqueous phase was further extracted with $\mathrm{CH}_{2} \mathrm{Cl}_{2}(4 \times 10$ $\mathrm{mL})$ and the combined organic phases were washed with $\mathrm{H}_{2} \mathrm{O}(30 \mathrm{~mL})$, dried over $\mathrm{Na}_{2} \mathrm{SO}_{4}$ and evaporated. The residue was purified by column chromatography (hexane/ $\mathrm{CH}_{2} \mathrm{Cl}_{2}, 8: 2$ ) affording DFE (44\%), TrFE (27\%) and TFE (8\%).

\section{Acknowledgements}

This work was supported by the University of Milan, the National Research Council and Regione Lombardia-FondazioneCARIPLO "SmartMatLab Centre" Project. The authors thank Mr. A. Figini for some EIS measures.

Keywords: alkenes • heterocycles • electrochemistry • electrooligomerization $\cdot$ solid state emission

[1] R. Hu, N. L. C. Leung, B. Z. Tang, Chem. Soc. Rev. 2014, 43, $4494-4562$.

[2] J. Luo, Z. Xie, J. W. Y. Lam, L. Cheng, H. Chen, C. Qiu, H. S Kwok, X. Zhan, Y. Liu, D. Zhu, B. Z. Tang, Chem. Commun., 2001, $1740-1741$.

[3] a) A. Qin, B. Z. Tang in Aggregation-Induced Emission: Fundamentals and Applications, Vol. 1 - 2, John Wiley \& Sons Ltd, London, 2008; b) J. Mei, N. L. Leung, R. T. Kwok, J. W. Lam, B. Z. Tang, Chem. Rev., 2015, 115, 11718 - 11940. c) R. T. Kwok, C. W. Leung, J. W. Lam, B. Z. Tang, Chem. Soc. Rev., 2015, 44, 42284238.

[4] Z. Zhao, J. W. Y. Lam, B. Z. Tang, J. Mater. Chem. 2012, 22, $23726-23740$.

[5] N. Xie, Y. Liu, R. Hu, N. L. C. Leung, M. Arseneault and B. Z. Tang, Isr. J. Chem., 2014, 54, 958 - 966.

[6] A. Bolzoni, L. Viglianti, A. Bossi, P. R. Mussini, S. Cauteruccio, C. Baldoli, E. Licandro, Eur. J. Org. Chem., 2013, 7489 - 7499.

[7] E. Castagnino, Tetrahedron Lett., 1985, 26, 6399 - 6402.

[8] T. Suzuki, H. Shiohara, M. Monobe, T. Sakimura, S. Tanaka, Y. Yamashita, T. Miyashi, Angew. Chem. 1992, 104, 454 - 456; Angew. Chem. Int. Ed. 1992, 31, 455 - 458.

[9] Electrochemical and photophysical studies on TFE have been presented in a poster communication: L. Viglianti, P.R. Mussini, C Baldoli, E. Licandro et al., "65th Annual Meeting of the International Society of Electrochemistry" 31st August-5th September 2014, Lausanne, Switzerland.

[10] B. K. Reddy, S. C. Gadekar, V. G. Anand, Chem. Commun., 2016, 52, $3007-3009$.

[11] P. Lucas, N. El Mehdi, H. A. Ho, D. Bélanger, L. Breau, Synthesis, 2000, $1253-1258$.

[12] M. L. N. Rao, D. N. Jadhav, P. Dasgupta, Org. Lett., 2010, 12, $2048-2051$.

[13] a) W.-Y. Wong, X.-Z. Wang, Z. He, A.-B. Djurisić, C.-T. Yip, K.-Y. Cheung, H. Wang, C.-S. Mak, W.-K. Chan, Nat. Mater., 2007, 6 $521-527$; b) R.S. Ashraf, M. Shahid, E. Klemm, M. Al-lbrahim S. Sensfuss, Macromol. Rapid Commun., 2006, 27, 1454 - 1459.

[14] S. Trasatti, Pure Appl. Chem. 1986, 58, 955 - 966.
[15] a) K. Meerholz, J. Heinze, Electrochim. Acta 1996, 41, 1839 1854. b) O. Gidron, Y. Diskin-Posner, M. Bendikov, J. Am. Chem. Soc. 2010, 132, $2148-2150$.

[16] a) S. Tanaka, M. Sato, K. Kaeriyama, Makromol. Chem. 1985, 186, 1685 - 1694; b) M. Bragadin, P. Cescon, A. Berlin, F. Sannicolò, Makromol. Chem. 1987, 188, 1425 - 1430; c) M. Martinez, J.R. Reynolds, S. Basak, D. A. Black, D. S. Marynick, M. Pomerantz, J. Pol. Sci. Part B: Polym. Phys 1988, 26, 911 - 920; d) J. O. Zerbino, W. J. Plieth, G. Kossmehl, J. Appl. Electrochem. 1991, 21, 935 940; e) M. Onoda, T. Iwasa, T. Kawai, K. Yoshino, J. Phys. D: Appl. Phys. 1991, 24, 2076 - 2083.

[17] a) G. Kossmehl, T. Greczmiel, Makromol. Chem. 1992, 193, 335 339; b) G. Kossmehl, T. Greczmiel, W. Plieth, Macromol. Chem Phys. 1994, 195, 3655 - 3664; c) A. Benahmed-Gasmi, P. Frère, J. Roncali, J. Electroan. Chem. 1996, 406, 231 - 234.

[18] M. M. Musiani, Electrochim. Acta 1990, 35, 1665 - 1670.

[19] a) An extensive study about the AIE behavior of thiophene and furan substituted ethylenes has been done in prof Tang's laboratories and is object of manuscripts in preparation. b) AIE properties and applications of TTE and TFE have been object of patent application: B.Z. Tang, L. Viglianti, N. Xie provisional patent application number: 62/386,380 (filed on: 30 November 2015); PCT application number: PCT/CN2016/086980 (filed on: June 24th 2016).

[20] a) U. Mitschke, P. Bäuerle, J. Mater. Chem. 2000, 10, 1471 1507; b) C. Botta, G. Patrinoiu, P. Picouet, S. Yunus, J.-E. Communal, F. Cordella, F. Quochi, A. Mura, G. Bongiovanni, M. Pasini, S. Destri, G. Di Silvestro Adv. Mater. 2004, 16, 1716 1721.

[21] Gaussian 09, Revision A.02, M. J. Frisch, G. W. Trucks, H. B. Schlegel, G. E. Scuseria, M. A. Robb, J. R. Cheeseman, G. Scalmani, V. Barone, B. Mennucci, G. A. Petersson, H. Nakatsuji, M. Caricato, X. Li, H. P. Hratchian, A. F. Izmaylov, J. Bloino, G. Zheng, J. L. Sonnenberg, M. Hada, M. Ehara, K. Toyota, R. Fukuda, J. Hasegawa, M. Ishida, T. Nakajima, Y. Honda, O. Kitao, H. Nakai, T. Vreven, J. A. Montgomery, Jr., J. E. Peralta, F. Ogliaro, M. Bearpark, J. J. Heyd, E. Brothers, K. N. Kudin, V. N. Staroverov, R. Kobayashi, J. Normand, K. Raghavachari, A Rendell, J. C. Burant, S. S. lyengar, J. Tomasi, M. Cossi, N. Rega, J. M. Millam, M. Klene, J. E. Knox, J. B. Cross, V. Bakken, C. Adamo, J. Jaramillo, R. Gomperts, R. E. Stratmann, O. Yazyev, A. J. Austin, R. Cammi, C. Pomelli, J. W. Ochterski, R. L. Martin, K. Morokuma, V. G. Zakrzewski, G. A. Voth, P. Salvador, J. J. Dannenberg, S. Dapprich, A. D. Daniels, Ö. Farkas, J. B. Foresman, J. V. Ortiz, J. Cioslowski, D. J. Fox, Gaussian, Inc., Wallingford CT, 2009.

[22] Y. Zhao, D. G. Truhlar, Theor. Chem. Accoun. 2008, 120, 215 241.

[23] R. Ditchfield, W. J. Hehre, J. A. Pople, J. Chem. Phys. 1971, 54, $724-728$.

[24] R. Krishnan, J. S. Binkley, R. Seeger, J. A. Pople, J. Chem. Phys. 1980, 72, $650-654$.

[25] See ref. 9; CCDC JOSKAD contains the supplementary crystallographic data for this paper. These data can be obtained free of charge from The Cambridge Crystallographic Data Centre via www.ccdc.cam.ac.uk/data_request/cif.

[26] a) G. Zhang, C. B. Musgrave, J. Phys. Chem. A 2007, 111, 1554 1561; b) L. Kronik, T. Stein, S. Refaely-Abramson, R. Baer, J. Chem. Theory Comput. 2012, 8, $1515-1531$.

[27] J. Cioslowski, J. Am. Chem. Soc. 1989, 111, 8333 - 8336.

[28] R. F. W. Bader in Atoms in Molecules. A Quantum Theory, Oxford University Press, Oxford, 1994.

[29] A. J. Bard, L. R. Faulkner in Electrochemical Methods. Fundamentals and Applications, 2nd ed. Wiley-VCH, New York, 2001, p. 648. 
[30] a) G. Gritzner, J. Kuta, Pure Appl. Chem. 1984, 56, 461 - 466; b) G. Gritzner, Pure Appl. Chem. 1990, 62, 1839 - 1858.

[31] J. Moreau, U. Giovanella, J.-P. Bombenger, W. Porzio, V. Vohra, L. Spadacini, G. Di Silvestro, L. Barba, G. Arrighetti, S. Destri, M. Pasini, M. Saba, F. Quochi, A. Mura, G. Bongiovanni, M. Fiorini, M. Uslenghi, C. Botta, ChemPhysChem 2009, 10, 647 - 653. 
Entry for the Table of Contents (Please choose one layout)

Layout 1:

\section{FULL PAPER}

A detailed electrochemical and photophysical comparative study of three parallel series of phenyl, thienyl and furyl substituted ethylenes has been carried out, showing the relationship between molecular structure (number and type of aromatic rings) and important functional properties.

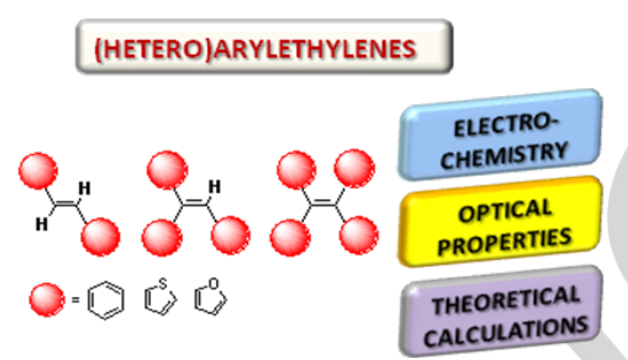

Lucia Viglianti, Francesca VillafioritaMonteleone, Chiara Botta, Patrizia R. Mussini, Emanuele Ortoleva, Silvia Cauteruccio, Emanuela Licandro and Clara Baldoli *

Page No. - Page No.

A comparative study of electrochemical, spectroscopic and structural properties of phenyl, thienyl and furyl substituted ethylenes

Layout 2:

\section{FULL PAPER}

((Insert TOC Graphic here; max. width: $11.5 \mathrm{~cm}$; max. height: $2.5 \mathrm{~cm})$ )
Author(s), Corresponding Author(s)*

Page No. - Page No.

Title 
